\title{
基于渐变凹模圆角半径的高强钢扭曲回弹补偿*
}

\author{
谢延敏 张飞王子豪 黄仁勇 杨俊峰 胡云川 \\ (西南交通大学机械工程学院 成都 610031)
}

\begin{abstract}
摘要: 为减少高强钢冲压成形扭曲回弹, 提出一种基于渐变凹模圆角半径的模具补偿方法。以高强钢 TRIP780 双 C 件为研究 对象, 采用板料冲压成形仿真软件 DYNAFORM 对该双 C 件的冲压、扭曲回弹过程进行数值模拟。提出一种评价双 C 件扭 曲回弹程度的指标, 并进行双 C 件扭曲回弹试验, 通过三坐标测量仪测量扭曲回弹角, 对有限元模型进行了验证。以冲压成 形后的扭曲回弹为优化目标, 结合相关的工艺参数, 利用 BP 神经网络, 基于正交试验, 建立凹模圆角半径渐变量、工艺参 数与扭曲回弹角之间的网络模型。最后采用遗传算法对该模型迭代寻优获得最优凹模圆角半径渐变量和工艺参数。通过对比 优化前后的扭曲回弹角, 证明了优化流程有效地减少了双 C 件扭曲回弹。该方法为扭曲回弹的控制提供了一种新的思路。 关键词: 高强钢; 扭曲回弹；渐变凹模圆角半径；BP 神经网络；遗传算法

中图分类号: TG156

\section{Compensation of Twist Springback in High-strength Steel Based on Gradient Die Radius}

\begin{abstract}
XIE Yanmin ZHANG Fei WANG Zihao HUANG Renyong YANG Junfeng HU Yunchuan
\end{abstract}
(School of Mechanical Engineering, Southwest Jiaotong University, Chengdu 610031)

\begin{abstract}
In order to reduce the twist springback appearing after the stamping of high-strength steel, a compensation method with gradient die radius is proposed. The double C rail of TRIP780 high-strength steel is taken as the research object. The sheet metal stamping simulation software DYNAFORM is used to numerically simulate the stamping and twist springback processes of the double $\mathrm{C}$ rail. An index to evaluate the twist springback of the double $\mathrm{C}$ rail is proposed. The experiment of twist springback for the double $\mathrm{C}$ rail is carried out. The twist springback angle is measured by means of a three-coordinate measuring instrument, and the finite element model is validated. The twist springback appearing after stamping is taken as the optimization target, and the related process parameters are taken into account. BP neural network is used to establish the network model between the gradient variation of die radius, process parameters and the twist springback angle based on orthogonal test. Finally, the model is iteratively optimized using genetic algorithm to obtain the optimum gradient variation of die radius and process parameters. The twist springback angle is compared before and after the optimization, which proves the optimization flow efficient to reduce the twist springback of the double $\mathrm{C}$ rail. This method provides a new way for the control of the twist springback.
\end{abstract}

Key words: high-strength steel; twist springback; gradient die radius; BP neural network; genetic algorithm

\section{0 前言}

汽车轻量化是当今汽车制造业的一种发展趋 势, 为了保证和提升汽车的安全性能, 高强度钢越 来越多的应用于汽车车身的覆盖件 ${ }^{[1]}$ 。然而高强度 钢具有较高的屈服强度, 在循环加载中出现包申格 效应会加重回弹的产生 ${ }^{[2]}$, 因此高强度钢比普通钢 的回弹问题更加突出。

近年来，由于高强钢冲压件不规则形状导致的

* 国家自然科学基金(51005193)和国家大学生创新性实验计划 (201710613033)资助项目。20180131 收到初稿, 20180718 收到修改稿
扭曲回弹问题越来越多, 国内外学者对扭曲回弹做 了很多研究。XUE 等 ${ }^{[3]}$ 分析了双相钢 C 形梁在冲压 过程中的扭曲回弹特性, 研究了 C 形梁扭曲回弹产 生的机理。郭超群等 ${ }^{[4]}$ 研究了开放式和半封闭式两 种 S 梁冲压件复杂回弹导致的扭曲回弹特性, 并分 析了压边力、摩擦、轧制方向对扭曲回弹的影响。 黄仁勇等 ${ }^{[5]}$ 基于双 $\mathrm{C}$ 梁试验的扭曲回弹角反求出其 混合硬化模型, 并进行了正交试验确定各相关因素 对扭曲回弹的的影响大小。吴否等 ${ }^{[6]}$ 侧重研究了板 料性能参数对扭曲回弹的影响, 得到了低屈服强度、 高应变硬化指数、高摩擦因数以及偏厚的材料可以 减弱扭曲回弹趋势的结论。 
为了减少扭曲回弹, 部分学者基于代理模型的 思想对冲压件的工艺参数进行了优化。谢延敏等 ${ }^{[7]}$ 通过正交试验篎选出对扭曲回弹影响较大的工艺参 数作为影响因素, 运用改进粒子群算法对某弯曲梁 进行优化, 有效的减少了弯曲梁的扭曲回弹。徐正 兴等 ${ }^{[8]}$ 结合均匀试验设计, 用二次多项式拟合出某 汽车结构件扭曲回弹角与工艺参数之间的回归模 型, 采用拉格朗日乘子算法对工艺参数进行了优化。 刘强等 ${ }^{[9]}$ 采用 DTF 法建立了三维非规则冲压件数值 模拟相对扭转角偏差与实际相对扭转角偏差之间的 传递函数，应用于模具型面的补偿，有效的控制了 扭转误差。

为了研究减小扭曲回弹的方法, 本文以双 C 件 为研究对象, 采用有限元分析软件 DYNAFORM, 通过试验验证了有限元模型, 并结合正交试验, 渐 变凹模圆角半径, BP 神经网络和遗传算法等方法, 以扭曲回弹为目标对其凹模圆角半径和工艺参数进 行优化。

\section{1 双 C 件有限元模型的建立}

\section{1 双 C 件几何尺寸}

$\mathrm{S}$ 梁类零件在实际冲压时会在法兰面和侧壁产 生的残余应力, 残余应力的不均匀分布在宏观上会 表现为一对扭曲力矩, 导致零件一端相对于另一端 扭转 ${ }^{[10]}$ 。为了更好的研究扭曲回弹现象, 设计了双 $\mathrm{C}$ 件, 中性层尺寸 $(\mathrm{mm})$ 如图 1 所示 ${ }^{[5]}$ 。

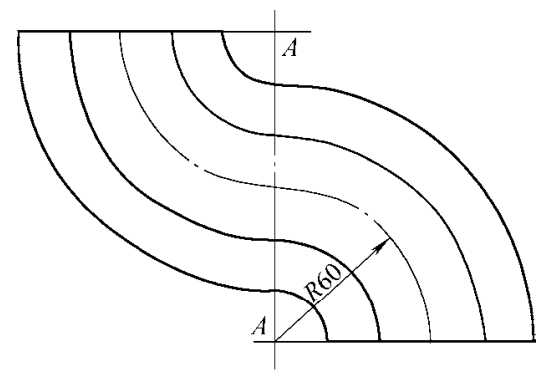

(a) 俯视图

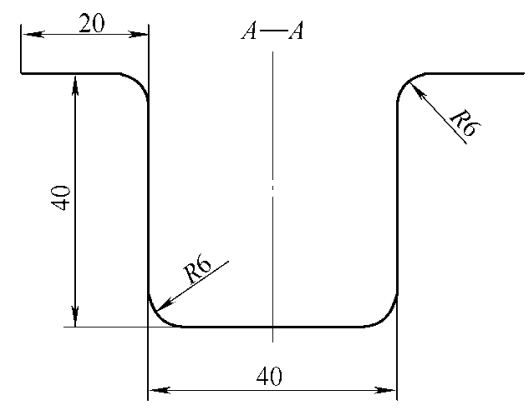

(b) $A-A$ 截面图

图 1 双 C 件中性层尺寸

\section{2 本构模型}

在进行双 C 件冲压、回弹数值模拟时, 采用 Barlat 模型 ${ }^{[11]}$, 屈服函数可表示为

$$
f=a\left|K_{1}+K_{2}\right|^{M}+a\left|K_{1}-K_{2}\right|^{M}+c\left|2 K_{2}\right|^{M}=2 \bar{\sigma}^{M}
$$

式中 $M-$ Barlat 指数;

$\bar{\sigma}$ 一单轴拉伸屈服应力一致的等效屈服应力;

$K_{1}, K_{2}$ 一应力张量不变量;

$$
\begin{aligned}
& K_{1}=\frac{\sigma_{x x}+h \sigma_{y y}}{2} ; \\
& K_{2}=\sqrt{\left(\frac{\sigma_{x x}-h \sigma_{y y}}{2}\right)^{2}+p^{2} \sigma_{x y}^{2}} ;
\end{aligned}
$$

$a, h, p$ 一材料常数;

$$
\begin{aligned}
& a=2-c=2-2 \sqrt{\frac{R_{0}}{1+R_{0}} \times \frac{R_{90}}{1+R_{90}}} ; \\
& h=\sqrt{\frac{R_{0}}{1+R_{0}} \times \frac{1+R_{90}}{R_{90}}} ;
\end{aligned}
$$

$R_{0}, R_{90}$ 一各向异性系数;

$$
p=\frac{\bar{\sigma}}{\tau_{\mathrm{s} 1}}\left(\frac{2}{2 a+2^{M} c}\right)^{1 / M} ;
$$

$\tau_{\mathrm{s} 1}$ ——纯剪切变形时的屈服切应力。

\section{3 有限元模型}

板料形状对冲压成形结果有较大的影响, 合理 的板料形状不仅可以减少冲压成形过程中的开裂、 起皱等缺陷, 还可以减少修边的困难。基于双 $\mathrm{C}$ 件 中性层形状反求出的板料形状如图 2 所示。

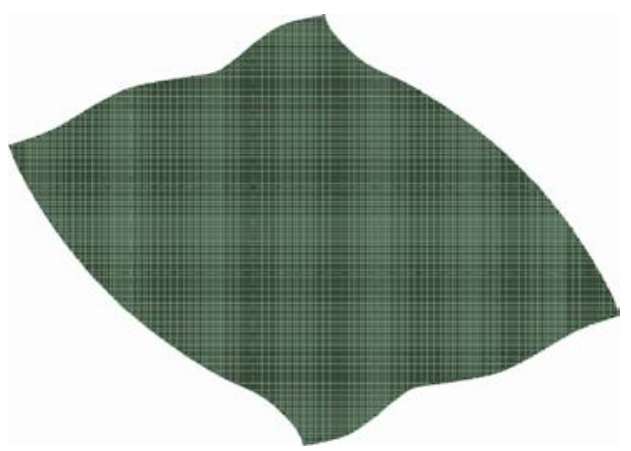

图 2 反求的板料形状

采用 DYNAFORM 对冲压、回弹过程进行数 值模拟, 有限元模型如图 3 所示。冲压过程采用动 力显示算法, 回弹过程采用静力隐式算法。板坏 材料采用 TRIP780 高强钢, 厚度为 $1 \mathrm{~mm}$, 板料的 单元为 $\mathrm{BT}$ 壳单元, 压边力为 $200 \mathrm{kN}$, 冲压行程 为 $40 \mathrm{~mm}$, 摩擦因数为 0.2 。相应的材料参数如表 1 所示 ${ }^{[12]}$ 。 


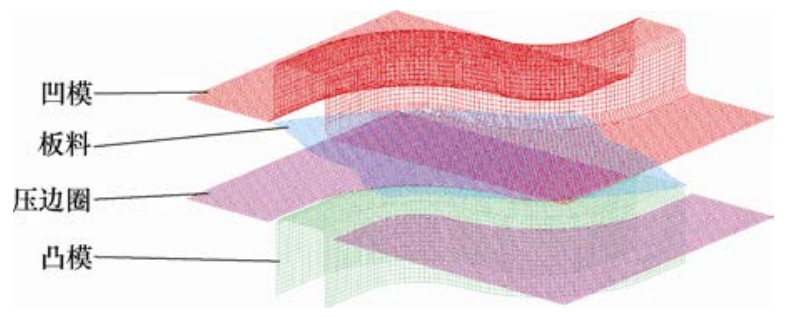

图 3 双 C 件有限元模型

表 1 材料参数

\begin{tabular}{|c|c|c|c|c|c|c|}
\hline \multirow{2}{*}{$\begin{array}{c}\text { 屈服强度 } \\
\text { /MPa }\end{array}$} & \multirow{2}{*}{$\begin{array}{c}\text { 弹性模量 } \\
\text { /GPa }\end{array}$} & \multirow{2}{*}{$\begin{array}{c}\text { 硬化指数 } \\
n\end{array}$} & \multirow{2}{*}{$\begin{array}{l}\text { Barlat } \\
\text { 指数 } M\end{array}$} & \multicolumn{3}{|c|}{ 各项异性指数 } \\
\hline & & & & $R_{0}$ & $R_{45}$ & $R_{90}$ \\
\hline 442.44 & 207 & 0.21 & 6.0 & 0.9 & 0.9 & 0.9 \\
\hline
\end{tabular}

\section{2 双 C 件试验及模型验证}

\section{1 扭曲回弹评价指标}

为了对双 $\mathrm{C}$ 件的扭曲回弹程度进行评价, 需要 对扭曲回弹角进行进行定量计算。由于冲压成形后 双 C 件 U 形截面底部为直线, 所以提出一种异面矢 量夹角代表扭曲回弹角的方法, 如图 4 所示。

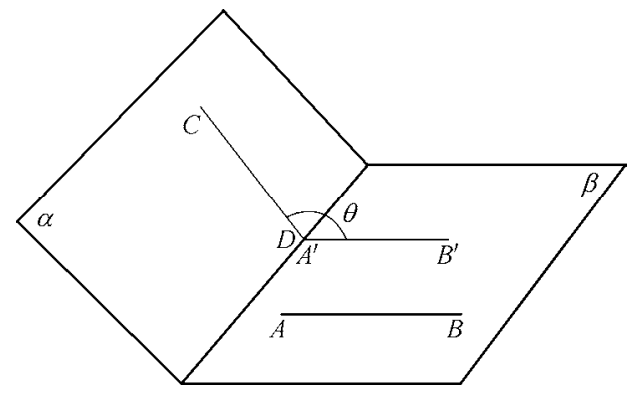

图 4 两异面矢量夹角

图 4 中 $A B$ 和 $C D$ 为两异面矢量, $A^{\prime} B^{\prime}$ 是 $A B$ 的平 行矢量, $A^{\prime}$ 点与 $D$ 点重合, $A^{\prime} B^{\prime}$ 与 $C D$ 夹角即为扭曲 回弹角。若已知 $\boldsymbol{A B}\left(x_{1}, y_{1}, z_{1}\right), \boldsymbol{C D}\left(x_{2}, y_{2}, z_{2}\right)$, 可得

$$
\theta=\arccos \frac{A B \cdot C D}{|A B| \cdot|C D|}
$$

\section{2 双 C 件冲压试验}

对 TRIP780 钢板采用线切割的方式加工出板料 形状, 冲压成形后双 C 件如图 5 所示。

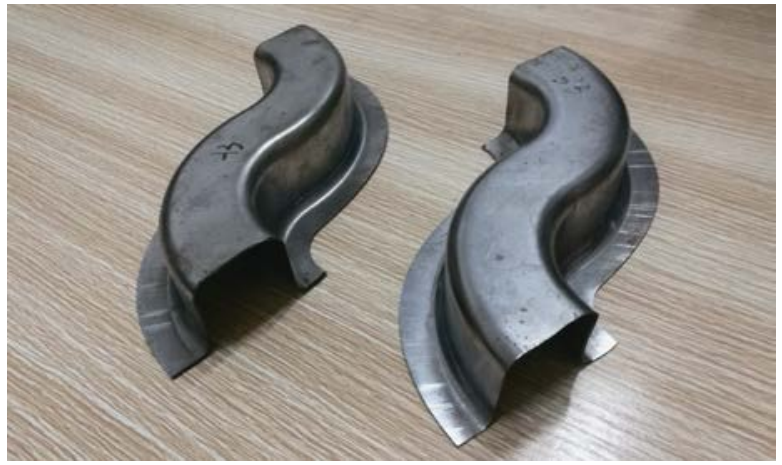

图 5 冲压成形的双 C 件
由于扭曲回弹是每个截面扭转累加的结果, 所以双 C 件前后两端扭转程度最大。以双 C 件两 端 $U$ 形截面底部直线所测夹角为评价双 $C$ 件扭曲 回弹指标。利用三坐标测量仪测量坐标, 测量点 位置如图 6 所示, $A B 、 C D$ 分别为两端底面直线 上两点。

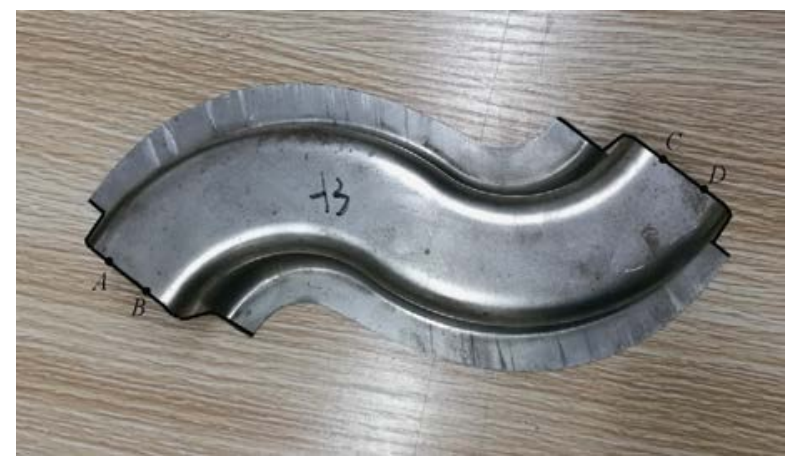

图 6 测量点位置

经计算得到双 $\mathrm{C}$ 件试验的扭曲回弹角平均值为 $1.428^{\circ}$, 经有限元模型测得扭曲回弹角平均值为 $1.304^{\circ}$, 误差大小为 $8.68 \%$, 验证了有限元模型具有 较高的合理性和可靠性, 可以代替物理试验对双 C 件扭曲回弹进行优化。

\section{3 扭曲回弹模具补偿及参数优化}

\section{1 基于渐变凹模圆角半径的模具补偿}

凹模圆角半径对于板料流动有重要影响。在冲 压过程中, 板料在凸模的作用下沿着凹模圆角半径 流动, 当凹模圆角半径较小时, 凹模进口处曲率较 大, 产生较大应力, 板料流动变困难。相反, 将凹 模圆角半径增大使得凹模进口曲率很小, 产生应力 较小, 板料流动变得更加流畅。

板料流动会影响成形后的双 $\mathrm{C}$ 件应力分布, 而 侧壁应力的不平衡是造成扭曲回弹的重要原因。所 以可以通过凹模圆角半径的变化来控制板料的流 动, 进而控制扭曲回弹 ${ }^{[3]}$ 。成形后双 $\mathrm{C}$ 件沿 $x$ 方向 位移如图 7 所示, 小弧面侧壁沿 $x$ 方向位移从中部 向两端逐渐减小, 而大弧面侧壁中部沿 $x$ 方向位移 则略小于两端。基于此提出了一种渐变凹模圆角半 径的模具补偿方法, 如图 8 所示。

将较大的圆角半径设置在产生位移较大的侧 壁处。大弧面处凹模圆角半径由两端向中间逐渐 变小, 小弧面处凹模圆角半径由两端向中间逐渐 变大, 可以减少板料流动的不平衡程度, 继而减 小扭曲回弹。 


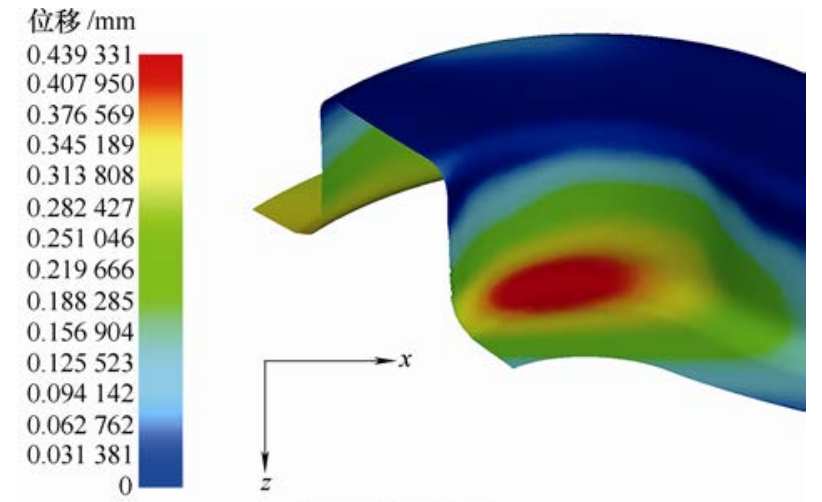

(a) 小弧面侧壁处

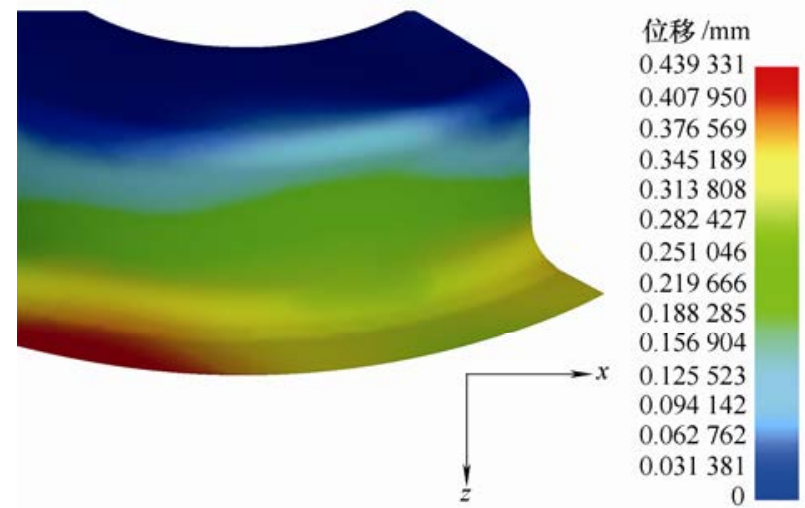

(b) 大弧面侧壁处

图 7 成形后沿 $x$ 方向位移

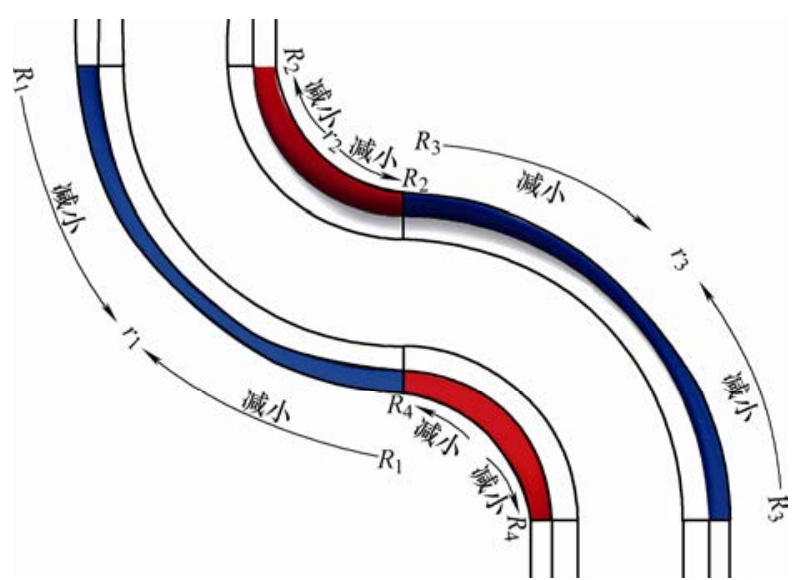

图 8 渐变凹模圆角半径模具补偿

对于大弧面、小弧面而言, 凹模圆角半径渐变 量可表示为

$$
\begin{array}{cc}
R_{i}-r_{i}=\Delta R_{i} & i=1,3 \\
r_{i}-R_{i}=\Delta R_{i} & i=2,4
\end{array}
$$

式(3)、(4)中, $R_{1} 、 R_{2} 、 R_{3} 、 R_{4}$ 为凹模初始圆角半径 值 $6 \mathrm{~mm}$ 。

将有限元模型的 4 个圆角半径依次渐变 $2 \mathrm{~mm}$, 并带入 DYNAFORM 仿真得到回弹模型, 测得此时 扭曲回弹角减小了 $0.421^{\circ}$, 验证了渐变凹模圆角半 径的模具补偿方法对减轻双 C 件扭曲回弹程度起到 了作用。

\section{2 正交试验}

为了减少有限元仿真的次数, 提高优化效率, 寻找最优的凹模圆角半径渐变量和工艺参数, 首先 利用正交试验设计方法安排试验 ${ }^{[13]}$ 。选取凹模圆角 半径渐变量 $\Delta R_{1} \sim \Delta R_{4}(\mathrm{~mm})$, 压边力 $F(\mathrm{kN})$, 冲压速 度 $v\left(\mathrm{~m} \cdot \mathrm{s}^{-1}\right)$, 摩擦因数 $\mu$ 作为试验因素, 以扭曲回 弹角 $\theta\left(^{\circ}\right)$ 作为试验指标, 试验因素与水平如表 2 所 示 ${ }^{[14]}$ 。试验选用 $L_{27}\left(3^{13}\right)$ 正交试验表，正交表如表 3 所示。

表 2 正交试验因素水平表

\begin{tabular}{cccccccc}
\hline \multirow{2}{*}{ 因素 } & \multicolumn{4}{c}{ 半径渐变量 $/ \mathrm{mm}$} & \multicolumn{2}{c}{ 力 $F /$} & \multicolumn{2}{c}{ 速度 $v /$} & 摩擦因数 \\
\cline { 2 - 5 } & $\Delta R_{1}$ & $\Delta R_{2}$ & $\Delta R_{3}$ & $\Delta R_{4}$ & $\mathrm{kN}$ & $\left(\mathrm{m} \cdot \mathrm{s}^{-1}\right)$ & $\mu$ \\
\hline 水平 1 & 0 & 0 & 0 & 0 & 200 & 2.5 & 0.100 \\
水平 2 & 1 & 1 & 1 & 1 & 250 & 3.5 & 0.115 \\
水平 3 & 2 & 2 & 2 & 2 & 300 & 4.5 & 0.125 \\
\hline
\end{tabular}

\begin{tabular}{|c|c|c|c|c|c|c|c|c|}
\hline \multirow{2}{*}{$\begin{array}{l}\text { 试验 } \\
\text { 序号 }\end{array}$} & \multicolumn{4}{|c|}{ 半径渐变量/mm } & \multirow{2}{*}{$\begin{array}{c}\text { 力 } F / \\
\mathrm{kN} \\
\end{array}$} & \multirow{2}{*}{$\begin{array}{l}\text { 速度 } v / \\
\left(\mathrm{m} \cdot \mathrm{s}^{-1}\right)\end{array}$} & \multirow{2}{*}{$\begin{array}{c}\text { 摩擦因数 } \\
\mu \\
\mu\end{array}$} & \multirow{2}{*}{$\begin{array}{c}\text { 回弹角度 } \theta \\
\quad /\left(^{\circ}\right)\end{array}$} \\
\hline & $\Delta R_{1}$ & $\Delta R_{2}$ & $\Delta R_{3}$ & $\Delta R_{4}$ & & & & \\
\hline 1 & 0 & 0 & 0 & 0 & 200 & 2.5 & 0.100 & 1.303 \\
\hline 2 & 0 & 0 & 0 & 0 & 250 & 3.5 & 0.115 & 0.837 \\
\hline 3 & 0 & 0 & 0 & 0 & 300 & 4.5 & 0.125 & 0.710 \\
\hline 4 & 0 & 1 & 1 & 1 & 200 & 2.5 & 0.100 & 1.597 \\
\hline 5 & 0 & 1 & 1 & 1 & 250 & 3.5 & 0.115 & 1.349 \\
\hline 6 & 0 & 1 & 1 & 1 & 300 & 4.5 & 0.125 & 0.703 \\
\hline 7 & 0 & 2 & 2 & 2 & 200 & 2.5 & 0.100 & 1.892 \\
\hline 8 & 0 & 2 & 2 & 2 & 250 & 3.5 & 0.115 & 1.713 \\
\hline 9 & 0 & 2 & 2 & 2 & 300 & 4.5 & 0.125 & 0.634 \\
\hline 10 & 1 & 0 & 1 & 2 & 200 & 3.5 & 0.125 & 1.531 \\
\hline 11 & 1 & 0 & 1 & 2 & 250 & 4.5 & 0.100 & 0.608 \\
\hline 12 & 1 & 0 & 1 & 2 & 300 & 2.5 & 0.115 & 2.686 \\
\hline 13 & 1 & 1 & 2 & 0 & 200 & 3.5 & 0.125 & 1.246 \\
\hline 14 & 1 & 1 & 2 & 0 & 250 & 4.5 & 0.100 & 0.407 \\
\hline 15 & 1 & 1 & 2 & 0 & 300 & 2.5 & 0.115 & 2.361 \\
\hline 16 & 1 & 2 & 0 & 1 & 200 & 3.5 & 0.125 & 1.745 \\
\hline 17 & 1 & 2 & 0 & 1 & 250 & 4.5 & 0.100 & 0.702 \\
\hline 18 & 1 & 2 & 0 & 1 & 300 & 2.5 & 0.115 & 2.764 \\
\hline 19 & 2 & 0 & 2 & 1 & 200 & 4.5 & 0.115 & 0.471 \\
\hline 20 & 2 & 0 & 2 & 1 & 250 & 2.5 & 0.125 & 2.551 \\
\hline 21 & 2 & 0 & 2 & 1 & 300 & 3.5 & 0.100 & 1.401 \\
\hline 22 & 2 & 1 & 0 & 2 & 200 & 4.5 & 0.115 & 0.511 \\
\hline 23 & 2 & 1 & 0 & 2 & 250 & 2.5 & 0.125 & 2.817 \\
\hline 24 & 2 & 1 & 0 & 2 & 300 & 3.5 & 0.100 & 1.530 \\
\hline 25 & 2 & 2 & 1 & 0 & 200 & 4.5 & 0.115 & 0.667 \\
\hline 26 & 2 & 2 & 1 & 0 & 250 & 2.5 & 0.125 & 2.671 \\
\hline 27 & 2 & 2 & 1 & 0 & 300 & 3.5 & 0.100 & 1.531 \\
\hline
\end{tabular}

表 3 正交试验设计表

采用极差分析法, 对试验结果分析可得, 各因 素对双 $\mathrm{C}$ 件扭曲回弹影响程度大小依次为: $v>\mu>\Delta R_{1}>F>\Delta R_{2}>\Delta R_{4}>\Delta R_{3}$ 。各因素水平对扭曲回弹 角的影响如图 9 所示。 


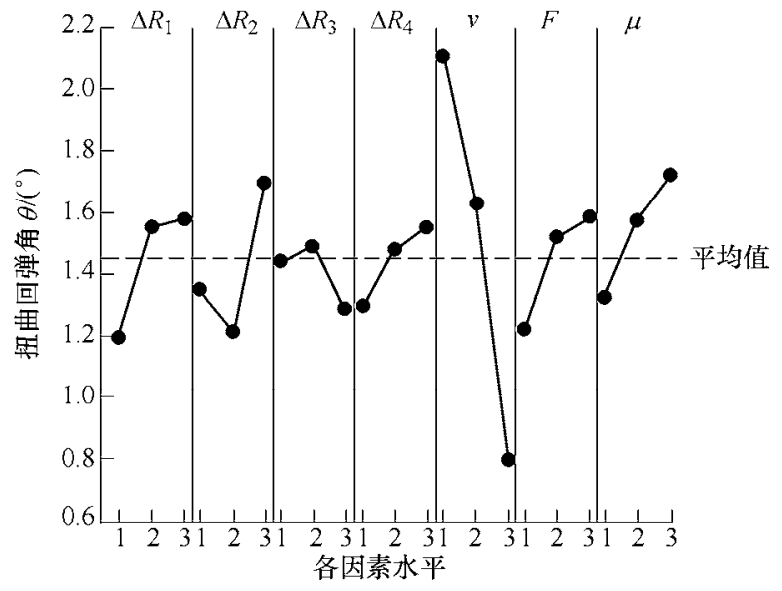

图 9 各因素水平对扭曲回弹角的影响

\subsection{BP 神经网络}

\subsubsection{BP 神经网络结构}

基于正交试验数据, 通过 BP 神经网络训练出 各因素与扭曲回弹角的映射关系。

BP 神经网络 (Back propagation neural network) 是一种并行多层前馈型网络, 通常一个三层神经网 络可以用 $\mathrm{S}$ 型传递函数根据需要的精度逼近任意连 续性函数 ${ }^{[15]}$, 其结构如图 10 所示。

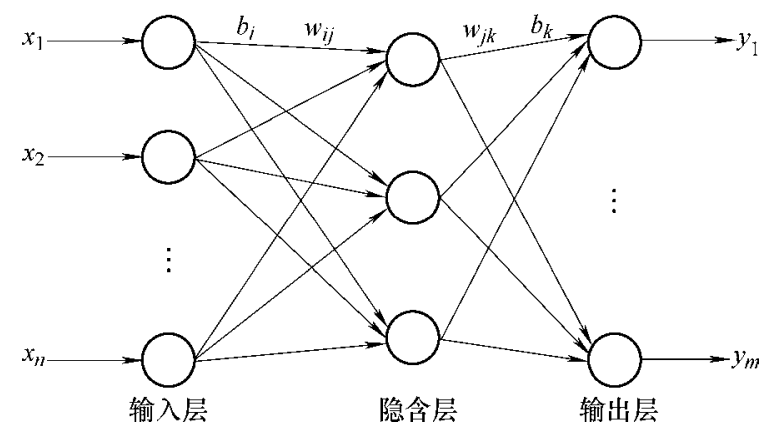

图 10 BP 神经网络结构

图 10 中, $x$ 为 BP 神经网络的输入, $y$ 为网络 的预测输出, $w$ 为网络权值, $b$ 为网络阈值。

模型设置含有 7 个输入节点, 4 个隐含层节点, 1 个输出节点。在构建网络模型时, 输入层与隐含 层传递函数为正切 $\mathrm{S}$ 型函数( $\tan \mathrm{sig}$ ), 隐含层与输出 层传递函数为线性传递函数(purelin), 其中训练方式 为 Levenberg-Marquardt 的 BP 算法训练函数 (trainlm) ${ }^{[16]}$ 。

设该网络的输入矢量为 $\boldsymbol{X}=\left(x_{1}, x_{2}, \cdots, x_{n}\right)^{\mathrm{T}}$, 则 在隐含层第 $j$ 个节点的输出为

$$
g_{j}(x)=\tan \operatorname{sig}\left(\sum_{i=1}^{n} w_{i j} x_{i}+b_{i}\right)
$$

式中 $w_{i j}$ 一第一层至第二层的权值;

$b_{i}$ 一一第二层的阈值;

$\tan \operatorname{sig}(x)=\frac{2}{1+\exp (-2 x)}-1$ 。
网络输出为

$$
y(x)=\sum_{j=1}^{p} w_{j k} g_{j}(x)+b_{k}
$$

式中 $p$ 一隐含层节点数;

$k$ 一一输出层节点数;

$w_{j k}$ 一一第二层至第三层的权值;

$b_{k}$ 一输出层阈值。

\subsubsection{BP 神经网络训练}

BP 算法是基于梯度下降的方法来调节网络的 连接权值, 通过网络预测输出与期望输出之间的误 差来反复修改网络的权值, 直至最终达到容差要求。

以正交试验设计法得出的 27 组数据作为训练 样本, 其中试验因素值为输入变量。基于 BP 神经 网络的参数优化流程如图 11 所示。

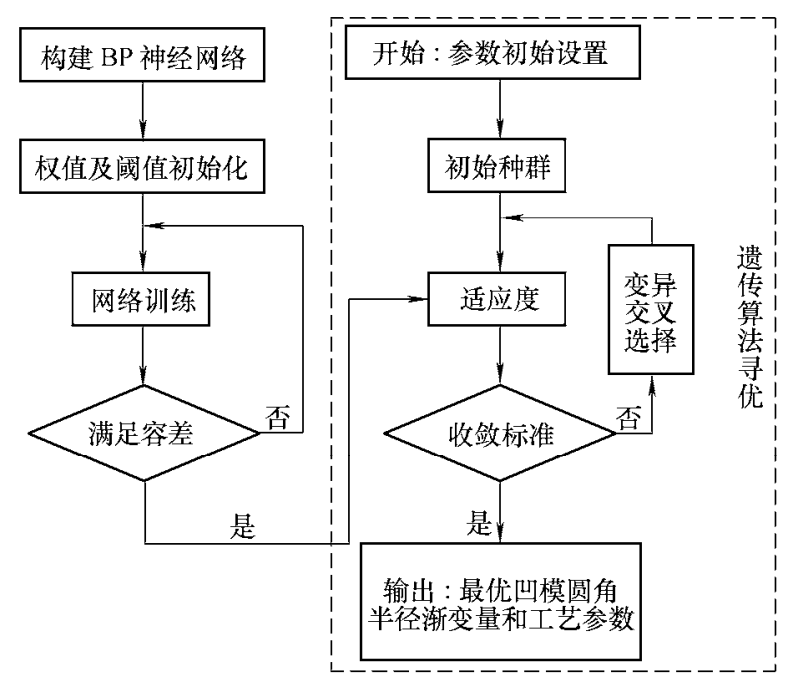

图 11 基于 BP 神经网络的参数优化流程

为了评估神经网络模型的可靠性, 在训练过程 中加入了方均误差(Mean-square error, MSE)评价指 标, 即方均误差值越小, 网络模型确定的映射关系 误差越小, 训练中结合方均误差曲线进行模型精度 分析 ${ }^{[17]}$ 。

经过多次训练, 得到模型误差值为 $3.32 \times 10^{-19}$, 模型在 9 步就可以收敛到要求精度，且最小验证方 均误差值在 12 步达到 0.008132 6, 说明网络模型精 度较高, 达到预期误差要求。方均误差曲线如图 12 所示。

\section{4 凹模圆角半径和工艺参数的优化}

基于凹模圆角渐变量和工艺参数建立代理模型 后, 需要对各参数进行寻优, 采用了遗传算法进行 最优值求解。

遗传算法基于自然选择的生物进化，是一种模 仿生物进化过程的随机方法，对一些非线性、多模 型、多目标的函数优化具有较好的效果。遗传算 


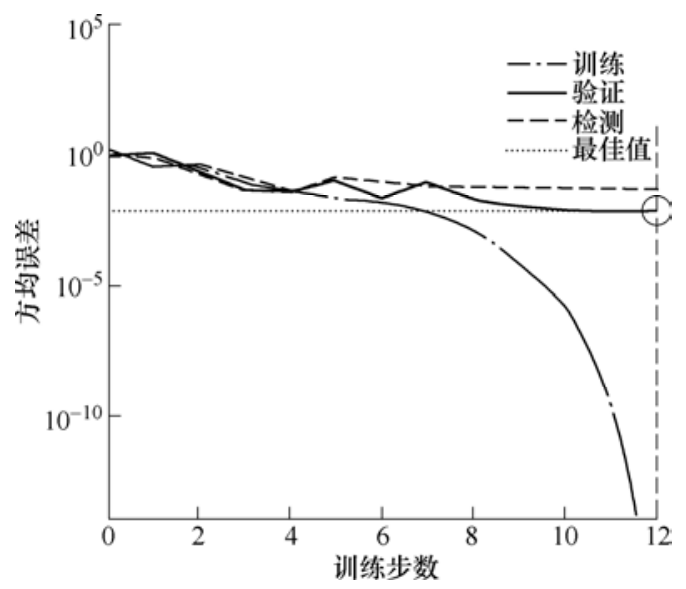

图 12 方均误差曲线图

法开始以初始种群为基础进行适应度检测, 满足 要求的种群直接输出, 而不满足的种群需要经过 选择、交叉、变异等过程循环检测直至满足要求 为止。

将最优的神经网络模型作为遗传算法适应度函 数, 以正交试验各因素的水平 1 与水平 3 构成的区 间为限制条件，通过遗传算法迭代寻优，获得模型 的最优值为 0.3086 , 适应度曲线如图 13 所示。此 时网络的输入为最优的凹模圆角半径渐变量和工艺 参数, 最优凹模圆角半径渐变量和工艺参数如表 4 所示。

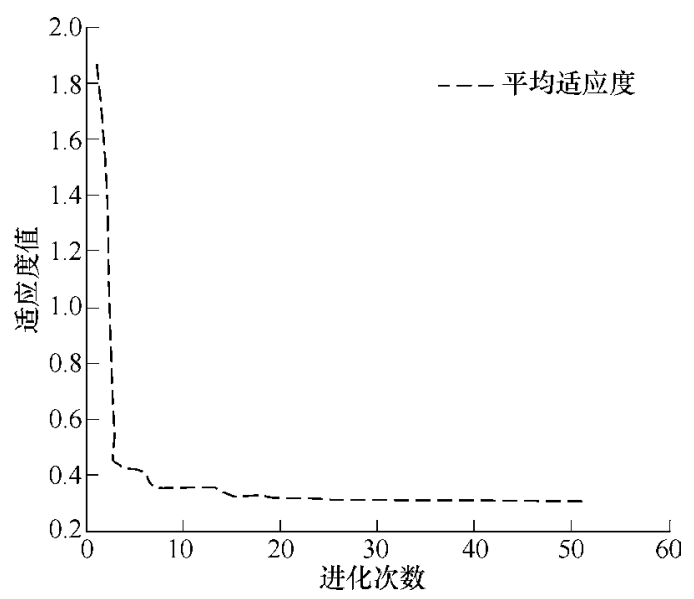

图 13 遗传算法适应度曲线

表 4 最优凹模圆角半径渐变量和工艺参数

\begin{tabular}{ccccccc}
\hline \multicolumn{5}{c}{ 半径渐变量 $/ \mathrm{mm}$} & & \\
\hline$\Delta R_{1}$ & $\Delta R_{2}$ & $\Delta R_{3}$ & $\Delta R_{4}$ & & 速度 $v /\left(\mathrm{m} \cdot \mathrm{s}^{-1}\right)$ & 摩擦因数 $\mu$ \\
\hline 0.0858 & 0 & 1.0439 & 0 & 224.2186 & 4.3169 & 0.1 \\
\hline
\end{tabular}

为了验证遗传算法寻优结果的准确性, 将寻优 得到的最优凹模圆角半径渐变量和工艺参数带入双 C 件有限元模型中检验。考虑到实际加工过程中的 精度, 将凹模圆角半径渐变量和工艺参数进行简化, 简化后的有限元模型相关参数如表 5 所示。
表 5 简化的最优凹模圆角半径渐变量和工艺参数

\begin{tabular}{ccccccc}
\hline \multicolumn{3}{c}{ 半径渐变量 $/ \mathrm{mm}$} & 力 & 速度 $v /$ \\
\cline { 1 - 3 }$\Delta R_{1}$ & $\Delta R_{2}$ & $\Delta R_{3}$ & $\Delta R_{4}$ & $F / \mathrm{kN}$ & $\begin{array}{c}\text { 摩擦因数 } \\
\left(\mathrm{m} \cdot \mathrm{s}^{-1}\right)\end{array}$ & $\mu$ \\
\hline 0.1 & 0 & 1.0 & 0 & 224 & 4.3 & 0.1 \\
\hline
\end{tabular}

在此参数下 $R_{1}$ 处的圆角半径从原来的 $6 \mathrm{~mm}$ 变 成由两端 $6 \mathrm{~mm}$ 向中间渐变为 $5.9 \mathrm{~mm}, R_{3}$ 处的圆角 半径从原来的 $6 \mathrm{~mm}$ 变成由两端 $6 \mathrm{~mm}$ 向中间渐变 为 $5 \mathrm{~mm}, R_{2} 、 R_{4}$ 处半径保持原来 $6 \mathrm{~mm}$ 恒定不变。 此时 DYNAFORM 仿真测得双 C 件扭曲回弹角为 $0.3407^{\circ}$, 与模型最优化值的相对误差在 $10.4 \%$ 左 右, 满足精度要求。将优化后得到的扭曲回弹角与 冲压试验参数得到的扭曲回弹角相比较可以看到, 扭曲回弹大大减小。

\section{4 结论}

(1) 对 TRIP780 高强钢双 C 件进行冲压试验, 验证了基于 Barlat 本构的有限元模型的准确性。

(2) 提出了渐变凹模圆角半径的模具补偿方法, 该方法减少了板料流动的不平衡程度, 从而减小双 C 件冲压后扭曲回弹角的大小。

（3）通过正交试验设计，得到了各试验因素对 扭曲回弹影响程度; 通过 BP 神经网络结合遗传算法 进行参数优化, 得到了最优的凹模圆角半径渐变量和 工艺参数。该方法可以有效减小双 $\mathrm{C}$ 件的扭曲回弹。

\section{参 考 文 献}

[1] ANDERSSON A. Numerical and experimental evaluation of springback in advanced high strength steel[J]. Journal of Materials Engineering and Performance, 2007, 16(3): 301-307.

[2] 胡康康, 彭雄奇, 陈军, 等. 基于 Yoshida-Uemori 材料 模型的汽车结构件冲压回弹分析 [J]. 材料科学与工艺, 2011, 19(6): 43-47.

HU Kangkang, PENG Xiongqi, CHEN Jun, et al. Springback prediction of automobile body panel based on Yoshida-Uemori material model[J]. Material Science \& Technology, 2011, 19(6): 43-47.

[3] XUE X, LIAO J, VINCZE G, et al. Twist springback characteristics of dual-phase steel sheet after non-axisymmetric deep drawing[J]. International Journal of Material Forming, 2015， 10(2): 267-278.

[4] 郭超群, 陈军, 陈劼实, 等. 超高强度薄板扭曲回弹特 性的数值模拟与试验分析 $[\mathrm{J}]$. 上海交通大学学报, 2010，44(4): 468-472.

GUO Chaoqun, CHEN Jun, CHEN Jieshi, et al. Numerical simulation and experimental validation of 
distortional springback of advanced high-strength steel sheet metal forming[J]. Journal of Shanghai Jiaotong University, 2010, 44(4): 468-472.

[5] 黄仁勇, 谢延敏, 唐维, 等. 基于混合硬化模型的 TRIP780 高强钢双 C 梁扭曲回弹仿真与试验 $[\mathrm{J}]$. 工程 设计学报, 2017, 24(6): 668-674.

HUANG Renyong, XIE Yanmin, TANG Wei, et al. Experiment and simulation on twist springback for the double C rail of TRIP780 high strength steel based on mixed hardening model[J]. Chinese Journal of Engineering Design, 2017, 24(6): 668-674.

[6] 吴否, 李光耀, 曹昭展, 等. 高强钢材料性能对汽车零 件扭曲回弹的影响 [J]. 塑性工程学报, 2009, 16(3): 13-17.

WU Lei, LI Guangyao, CAO Zhaozhan, et al. The effect of material properties influenced on torsion springback of high strength steel parts[J]. Journal of Plasticity Engineering, 2009, 16(3): 13-17.

[7] 谢延敏, 孙新强, 田银, 等. 基于改进粒子群算法和小 波神经网络的高强钢扭曲回弹工艺参数优化 $[\mathrm{J}]$. 机械 工程学报, 2016, 52(19): 162-167.

XIE Yanmin, SUN Xinqiang, TIAN Yin, et al. Optimization of parameters in twist springback process for high-strength sheets based on improved partical swarm optimization algorithm and neural network[J]. Journal of Mechanical Engineering, 2016， 52(19): 162-167.

[8] 徐正兴, 路平, 江开勇. 高强度钢冲压成形中扭曲回弹 仿真与工艺参数优化 $[\mathrm{J}]$. 机械设计与制造, 2012(10): 262-264.

XU Zhengxing, LU Ping, JIANG Kaiyong. Torsion springback simulation and optimization of process parameters of high strength steel in the stamping forming [J]. Machinery Design \& Manufacture, 2012(10) : 262-264.

[9] 刘强, 阮锋, 薛新, 等. 三维非规则冲压件的回弹扭转 补偿控制[J]. 华南理工大学学报, 2009, 37(9): 93-97. LIU Qiang, RUAN Feng, XUE Xin, et al. Compensation control of springback torsion for 3D irregular stamping parts[J]. Journal of South China University of Technology, 2009, 37(9): 93-97.

[10] 李洪周. 先进高强度钢扭曲回弹研究[D]. 长沙: 湖南大 学, 2009.

LI Hongzhou. A study on twist springback of advance high strength steel[D]. Changsha: Hunan University,
2009.

[11] BARLAT F, LEGE D J, BREM J C. A six-component yield function for anisotropic materials[J]. International Journal of Plasticity, 1991, 7(7): 693-712.

[12] 张卿卿, 李大永, 彭颖红, 等. TRIP780 高强度钢板动 态力学特性的研究 [J]. 塑性工程学报, 2009, 16(6): 6-10.

ZHANG Qingqing, LI Dayong, PENG Yinghong, et al. Research on the dynamic mechanical characteristics of TRIP780 high strength steel sheets[J]. Journal of Plasticity Engineering, 2009, 16(6): 6-10.

[13] 林忠钦, 刘罡, 李淑慧, 等. 应用正交试验设计提高 U 形件的成形精度[J]. 机械工程学报, 2002, 38(3): 83-89. LIN Zhongqin, LIU Gang, LI Shuhui, et al. Application orthogonal experiment design in increasing dimensional accuracy of U-shaped parts[J]. Chinese Journal of Mechanical Engineering, 2002, 38(3): 83-89.

[14] 姜奎华. 冲压工艺与模具设计 $[M]$. 北京：机械工业出 版社, 2005 .

JIANG Kuihua. Pressing technology and die design[M]. Beijing: China Machine Press, 2005.

[15] INAMDAR M, DATE P P, NARASIMHAN K, et al. Development of an artificial neural network to predict springback in air vee bending[J]. International Jounal of Advanced Manufacturing Technology, 2000, 16(5): 376-381.

[16] 谢延敏, 唐维, 黄仁勇, 等. 基于 SA-RBF 神经网络的 冲压成形拉延筋优化 [J]. 西南交通大学学报, 2017, 52(5): 970-976, 993.

XIE Yanmin, TANG Wei, HUANG Renyong, et al. Drawbead optimisation in stamping using SA-RBF neural networks[J]. Journal of Southwest Jiaotong University, 2017, 52(5): 970-976, 993.

[17] 秦国华, 谢文斌, 王华敏. 基于神经网络与遗传算法的 刀具磨损检测与控制 $[\mathrm{J}]$. 光学精密工程, 2015，23(5): 1314-1321.

QIN Guohua, XIE Wenbin, WANG Huamin. Detection and control for tool wear based on neural network and genetic algorithm[J]. Optics and Precision Engineering, 2015, 23(5): 1314-1321.

作者简介: 谢延敏(通信作者), 男, 1975 年出生, 博士, 副教授, 硕士 研究生导师。主要研究方向为先进塑性加工技术仿真和稳健设计等。 E-mail: xie_yanmin@swjtu.edu.cn 